\title{
Reorientación del Programa de Localización de Casos de Tuberculosis. Región Metropolitana, 2006
}

\author{
FERNANDO TABILO P.* y CARLOS PEÑA M.**
}

Guidances for case-finding. National Tuberculosis Program. Chile, 2006

\section{Introducción}

El Programa de Control de la Tuberculosis (PCT) basa su accionar, en cuanto al diagnóstico de esta enfermedad, en dos supuestos:

1) Que los pacientes con síntomas respiratorios consultarán espontáneamente a los Servicios de Salud, y 2) Que los médicos se acordarán de solicitar dos baciloscopías a los que tengan tos productiva de más de 3 semanas de duración.

Así, la localización de casos de tuberculosis, también llamada búsqueda de casos o pesquisa, depende de la colaboración de los equipos de salud, especialmente del estamento médico.

Esta búsqueda de enfermos con tuberculosis prioriza los casos pulmonares bacilíferos, entre los cuales se encuentran los portadores de lesiones cavitarias que están cursando estados avanzados de la enfermedad y supone que eventualmente deberían consultar por sus síntomas en la Atención Primaria. Aquí se espera que los médicos descarten la tuberculosis como diagnóstico diferencial de una tos productiva de más de dos semanas de evolución.

Hay dos estrategias de tamizaje: la labor clínica que efectúa el médico en la consulta y la encuesta baciloscópica que realiza personal sanitario entrenado entre los asistentes que, por cualquier motivo, se hallan en las salas de espera y otras dependencias de los consultorios.

Para conocer la procedencia de las baciloscopías en los casos detectados de tuberculosis, durante el segundo semestre del año 2004, se entrevistó a todos los enfermos Koch (+) del Servicio de Salud Metropolitano Suroriente (SSMSO), durante la primera semana que si- guió a su diagnóstico, estableciendo dónde se habían solicitado sus baciloscopías y de este modo asegurado el diagnóstico de tuberculosis (Figura 1).

Según la Figura 1, es probable que la Norma actual del PCT de pedir 50 baciloscopías por mil consultas de adultos en la atención primaria, no esté apuntando al área donde se originan los nuevos enfermos, que parecen generarse más frecuentemente de los especialistas broncopulmonares, servicios de emergencia y médicos privados.

Además, en los últimos años se está asistiendo al creciente aumento de casos procedentes de grupos vulnerables o de riesgo. Se consideran como tales a las personas que tienen una mayor probabilidad de enfermar, ya sea por deterioro inmunitario, mayor exposición a fuentes infectantes o menor probabilidad de diagnóstico

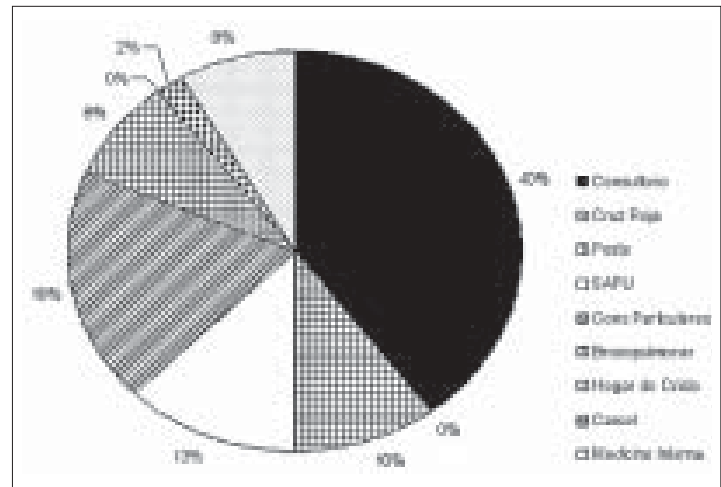

Figura 1. Distribución de la procedencia de las baciloscopías positivas en 47 casos. SAPU: Servicio de atención primaria de urgencia. Posta: Servicio de urgencia convencional.

\footnotetext{
* Servicio de Medicina, Hospital Sótero del Río y Programa de Control de la Tuberculosis, Servicio de Salud Metropolitano Sur-Oriente.

** Departamento Broncopulmonar, Hospital Clínico San Borja-Arriarán y Programa de Control de la Tuberculosis, Servicio de Salud Metropolitano Central.
} 
precoz o de acceso a la atención médica. Estos grupos fueron investigados inicialmente en el Servicio de Salud Metropolitano Central (SSMC) y luego se ampliaron a toda la Región Metropolitana.

Durante el período 2000-2003, en el SSMC, de un total de 833 casos pertenecientes a alguno de los grupos vulnerables, el 48,7\% tenía baciloscopías positivas. Las cifras correspondientes a años posteriores, fueron de $51,7 \%$ el año 2004 y $54,4 \%$ en 2005.

Para la Región Metropolitana, el porcentaje de grupos de riesgo varía según el Servicio entre 19,8 y $54,9 \%$, predominando en los adultos mayores (221 casos de los 971 informados el año 2005 , constituyendo el $28,8 \%$ de todas las tuberculosis bacilíferas).

Estos antecedentes nos muestran que debemos orientar la pesquisa desde una posición pasiva, donde habitualmente hay que practicar más de 200 baciloscopías para encontrar una positiva, a una pesquisa activa, dirigida a los grupos de donde proceden la mayoría de los pacientes contagiosos.

Resulta necesario, además, plantear una discusión en los equipos de tuberculosis del nivel intermedio, respecto a los cambios que deben efectuarse en los lugares donde se hacen actualmente más diagnósticos de tuberculosis y analizar una serie de situaciones que inciden en el rendimiento de la detección de casos:

\section{1) ¿Es en el nivel secundario donde deben concentrarse los esfuerzos de la pesquisa?}

Es importante conocer cuál es la proporción de pacientes tuberculosos derivados desde la Atención Primaria a los especialistas, la proporción de diagnósticos que se originan desde Servicios Broncopulmonares en consultas espontáneas y a partir de otros especialistas como infectólogos, internistas, etc. Esta información podría obtenerse en parte del libro de laboratorio de tuberculosis de cada Servicio, tanto a nivel local como intermedio.

\section{2) ¿Ya no hay motivación de los médicos por la pesquisa de la tuberculosis?}

Para conocer las motivaciones o interés de los profesionales y su relación en la disminución en el número de baciloscopias diagnósticas, se entrevistó a 16 médicos de atención de adultos de tres consultorios del SSMSO (90\%), con índices de pesquisa inferiores a 25 x 1.000, ubicados en sectores densamente poblados, con niveles superiores al $12 \%$ de cesantía (La Bandera, San Ramón y Silva Henríquez). Se pudo establecer un grado insuficiente de participación en la pesquisa de la tuberculosis, basado en la suposición de que la detección de esta enfermedad no constituía una prioridad, y que de alguna manera la búsqueda de casos no les competía directamente, como se pudo apreciar en la atención de urgencia, o existía la impresión de que la solicitud de baciloscopía era un instrumento demasiado complejo. Se hizo evidente que la voluntad comprometida del personal de salud debe ser estimulada.

\section{3) Desconocimiento de la tuberculosis y falta de incentivos en la práctica profesional}

En seminarios sobre Medicina Familiar se ha establecido que "la entrevista personal, apoyada con material escrito" es la acción más segura para modificar conductas en los médicos de esta especialidad. ¿Disponemos de tiempo y dinero para este tipo de supervisión personalizada? ¿Podría la tuberculosis resultar más interesante para ellos si conocieran sistemáticamente los resultados del programa local, datos históricos amenos, o bien al estimularlos a que propongan cambios en la aplicación de las estrategias locales, explicitando sus críticas? A los médicos se les debiera informar cuando su diagnóstico de tuberculosis fue exitoso, para reafirmar las conductas favorables a la pesquisa. Del mismo modo, debieran ser notificados de cuándo no se hizo el diagnóstico de tuberculosis, habiendo tenido la oportunidad de hacerlo.

\section{4) Reorientación del programa hacia las co- munidades organizadas.}

¿Deberíamos abandonar a los médicos de la atención primaria y contactar las unidades vecinales de las áreas de alto riesgo, para capacitarlas en pesquisa antituberculosa? Es frecuente que estas unidades tengan encargados de salud, pero su motivación es débil; responden bien ante situaciones de campaña, pero no a una situación de esfuerzo permanente, por lo que su alianza con la atención primaria es esencial.

\section{5) Consolidar el aporte del Estudio de Con- tactos}

Se debe dimensionar el real aporte del estudio de contactos, como estrategia en la localización de casos y el impacto preventivo que tiene la quimioprofilaxis, para establecer el número de casos evitados por esta acción. El seguimiento de la adherencia o cumplimiento de esta terapia preventiva también debe ser evaluado. Los análisis del SSMSO, demuestran un rendimiento del estudio de contactos, en el diagnóstico de nuevos 
Tabla 1. Estudio de contactos de pacientes tuberculosos. Servicio de Salud Metropolitano Sur Oriente 2005

\begin{tabular}{|c|c|c|c|c|c|c|c|c|}
\hline \multirow[t]{2}{*}{ Lugar } & \multicolumn{3}{|c|}{ Índices } & \multirow[t]{2}{*}{ Censados } & \multirow[t]{2}{*}{ Estudiados } & \multirow[t]{2}{*}{ No Estudiados } & \multirow[t]{2}{*}{ Nuevos Tb } & \multirow[t]{2}{*}{ Quimioprofilaxis } \\
\hline & $\mathbf{a}$ & $\mathbf{b}$ & c & & & & & \\
\hline \multicolumn{9}{|l|}{ San Ramón } \\
\hline San Ramón & 5 & 1 & & 24 & 23 & 1 & 1 & 6 \\
\hline La Bandera & 6 & 2 & & 29 & 29 & 0 & 0 & 9 \\
\hline \multicolumn{9}{|l|}{ La Granja } \\
\hline La Granja & 5 & & 2 & 8 & 8 & 0 & 0 & 0 \\
\hline Malaquías & 2 & & & 19 & 16 & 3 & 0 & 4 \\
\hline Granja Sur & 1 & 1 & & & & & & \\
\hline \multicolumn{9}{|l|}{ La Pintana } \\
\hline Santiago & 9 & 1 & 1 & 32 & 27 & 5 & 0 & 6 \\
\hline San Rafael & 4 & 1 & 1 & 26 & 25 & 1 & 1 & 8 \\
\hline El Roble & 4 & & & 13 & 12 & 1 & 0 & 0 \\
\hline Pablo de Rokha & 2 & & & 5 & 5 & 0 & 0 & 0 \\
\hline Flor Fernández & 4 & & 1 & 9 & 9 & 0 & 0 & 5 \\
\hline Santo Tomás & 1 & & & 4 & 4 & 0 & 0 & 0 \\
\hline \multicolumn{9}{|l|}{ La Florida } \\
\hline Los Quillayes & 4 & 2 & & 15 & 15 & 0 & 0 & 7 \\
\hline Villa O'Higgins & 3 & & & 11 & 10 & 0 & 0 & 2 \\
\hline Bellavista & 3 & 1 & & 4 & 4 & 0 & 0 & 0 \\
\hline Mafioletti & 7 & 1 & & 27 & 24 & 2 & 0 & 1 \\
\hline Los Castaños & 5 & & & 21 & 19 & 2 & 1 & 1 \\
\hline Santa Amalia & 2 & & & 8 & 8 & 0 & 0 & 0 \\
\hline \multicolumn{9}{|l|}{ Puente Alto } \\
\hline A del Río & 11 & 4 & & 51 & 49 & 2 & 2 & 15 \\
\hline San Jerónimo & 3 & & & 12 & 12 & & 1 & 1 \\
\hline Bernardo Leigthon & n 2 & & & 3 & 3 & & & \\
\hline Silva Henríquez & 6 & & 1 & 17 & 16 & 1 & 1 & 5 \\
\hline Padre Villaseca & 2 & & & 9 & 9 & & 1 & \\
\hline Vista Hermosa & 1 & & & 1 & 1 & & & \\
\hline Madre Teresa & 3 & & & 11 & 10 & 1 & & \\
\hline Karol Vojtila & 0 & & & & & & & \\
\hline \multicolumn{9}{|l|}{ San José de Maipo } \\
\hline San José & 0 & & & & & & & \\
\hline \multicolumn{9}{|l|}{ Pirque } \\
\hline Pirque & 1 & & & 7 & 7 & & & \\
\hline $\begin{array}{l}\text { Casos especiales } \\
\text { sin residencia }\end{array}$ & & & & & & & & \\
\hline demostrada & 2 & 1 & & 2 & 2 & & & \\
\hline Totales (n) & $88 * *$ & 15 & 6 & 358 & 339 & 19 & 8 & 70 \\
\hline Porcentajes & 100 & 17 & 7 & 100 & 95 & 5 & 2,3 & 21 \\
\hline
\end{tabular}

* Casos índices: a) pulmonares con baciloscopía (+); b) sin contactos; c) no estudiados. **82 casos con tuberculosis pulmonar baciloscopía $(+)$; 6 recaídas con tuberculosis pulmonar baciloscopía $(+)$. Nuevos $\mathrm{Tb}=$ casos nuevos.

casos de 2,3\%, y la indicación de terapia preventiva en el $25 \%$ de los estudiados (Tabla 1).

\section{6) Nuevos métodos diagnósticos mejorados}

Se advierte la necesidad de incorporar nuevas técnicas de diagnóstico, no sólo para mejorar la sensibilidad de los procedimientos actuales, sino también para reducir los tiempos diagnósticos y de los estudios de sensibilidad, y para conocer mejor la transmisión de la enfermedad mediante las técnicas de la epidemiología molecular.

7) Pesquisa focalizada a grupos de riesgo especiales

La pesquisa debe focalizarse en los actuales grupos de riesgo de hacer tuberculosis: adultos mayores de 75 años en hogares de ancianos, 
contactos íntimos de casos pulmonares bacilíferos, pacientes VIH (+) en programas de control del SIDA, inmigrantes, reclusos, vagabundos de hospederías, drogadictos en "caletas", pueblos originarios, pacientes psiquiátricos internos en sanatorios, diabéticos en programas de control, pacientes EPOC en salas ERA-Enfermedad Respiratoria Aguda), personal de salud y unidades vecinales organizadas escogidas.

\section{8) Cumplimiento de pesquisa en poblaciones de riesgo:}

El mayor riesgo de tuberculosis se presenta en personas de la tercera edad (Figura 2) y en los contactos de casos pulmonares bacilíferos, por lo que las actividades de localización de casos en estas poblaciones deberían tener una especial prioridad y un mejor control.

\section{9)Desconocimiento de la población general}

Necesidad de publicidad inteligente para acceder al eventual tuberculoso pulmonar con un mensaje claro y breve, en los medios de comunicación masiva. Incorporación de información en la malla curricular de enseñanza básica, media y universitaria, especialmente en las carreras de la salud.

\section{0)Estructura y organización de los niveles in- termedios del PCT}

Los equipos bien constituidos, pueden ejercer una mejor vigilancia de la localización de casos y establecer directrices sólidas en la programación de las actividades que fortalecen y estimulan a los equipos locales y crean espacios de consolidación en las autoridades de los servicios. Capacidad de organización y prestigio, son la mejor carta de presentación para lograr estímulos de persuasión.

\section{1) Colaboración del extrasistema}

La colaboración del extrasistema de salud es trascendental para la pesquisa. Esta posibilidad necesariamente pasa por la capacitación y difusión de las Normas Nacionales del PCT, para intensificar la colaboración público-privada.

\section{2) La realidad de los hogares de ancianos}

En el sector de población que cubre el SSMSO, la mayoría de los hogares de ancianos son clandestinos y el número de pacientes tuberculosos que han proporcionado hasta la fecha es menor del $2 \%$ en los últimos tres años. Tenemos la impresión que los ancianos que buscamos no están en estos lugares. No es infrecuente que lleguen a los servicios de emergencias acompañados de terceros, en pésimas condiciones generales, a veces agonizando, con la tuberculosis como una más de sus comorbilidades descompensadoras.

Los octogenarios actuales, sobrevivieron a una época en que más del $80 \%$ de sus padres y abuelos estaban infectados por el bacilo de la tuberculosis y esta enfermedad no tenía tratamiento, Aunque muchos murieron, otros han mantenido al microorganismo en estado latente en focos residuales. La reactivación de estas lesiones representa el $95 \%$ de los casos de tuberculosis en la población anciana actual. Se sabe que los mayores de 75 años tienen 12 veces más posibilidades de hacer una tuberculosis que sus nietos.

Cuando un anciano llega a una casa de reposo puede ocurrir que al compartir el aire conta-

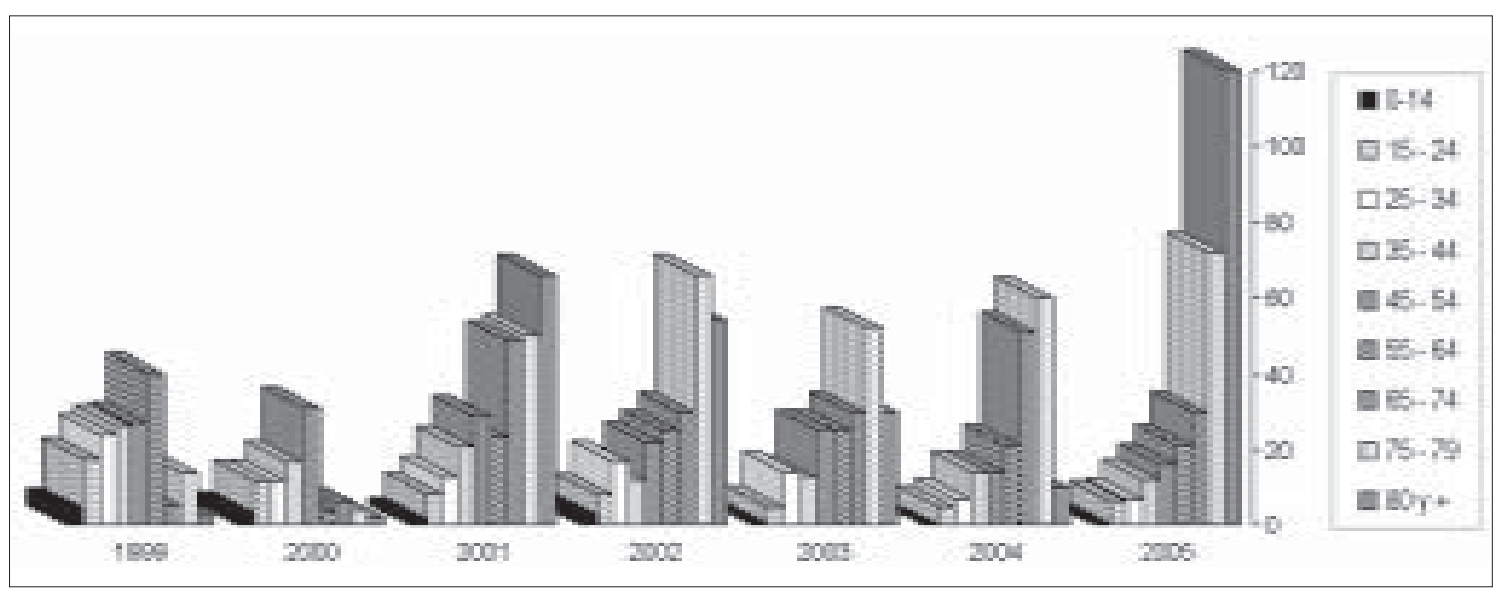

Figura 2. Riesgo de enfermar por Tuberculosis según grupos de edad (SSMSO). Tasas x 100.000. 
minado se infecte con otro anciano que tiene tuberculosis pulmonar y no lo sabe (en especial si no se tomó una radiografía de tórax a su ingreso); pero también pueden reactivarse sus propias lesiones latentes, porque se hizo diabético, tuvo una nutrición inadecuada, pasó por largos períodos de depresión, abusó del alcohol o, simplemente, porque se debilitó su inmunidad celular con los años.

No es necesario que un senescente tosa o expectore y menos aún que esté febril, para sospechar el comienzo de una tuberculosis; con frecuencia, el decaimiento persistente, la dorsalgia inespecífica, cambios de su humor y la descompensación de otra enfermedad (diabetes, EPOC, insuficiencia cardíaca o daño hepático crónico) deberían ponernos en alerta y decidirnos a tomar muestras de expectoración y una radiografía de tórax.

La tuberculosis en los ancianos puede comprometer otros órganos (glándulas suprarrenales, columna vertebral, pericardio, meninges y ganglios linfáticos). El examen físico frecuentemente no conduce al diagnóstico. La anamnesis puede ser improductiva por la falta de claridad mental e incluso la radiografía es fácilmente confundible con cáncer, neumonia o secuelas de otras patologías.

Durante el año 2005 ingresaron cuatro ancianos con tuberculosis desde casas de reposo, al servicio de urgencia del hospital Sótero del Río, en tan graves condiciones, que fallecieron antes de $48 \mathrm{~h}$ (dos de ellos tenían trastornos mentales). A ninguno se les había solicitado los exámenes bacteriológicos que les habrían salvado la vida y evitado el riesgo de contagio a sus compañeros.

\section{3) Los inmigrantes o extranjeros}

No se ha objetivado la tasa de multiresistencia en ellos, que parece ser más alta de lo esperado. Esta precisión puede provenir del Ins- tituto de Salud Pública si cumplimos con la norma de identificar las muestras con la condición de extranjero. La relación con los equipos similares en los países de procedencia, es un anhelo hasta ahora no logrado y constituye un desafío para nuestro sistema informático, ya que podría ser más expedito a través de Internet.

\section{4) La solicitud de baciloscopía}

El formato actual prioriza a los enfermos antes tratados que no han superado el $10 \%$ de todos los tuberculosos; en cambio, castiga el 90\% de los casos nuevos. Es necesario simplificar la orden, con un mínimo de datos, para hacerla atractiva a los médicos de urgencia. Mostramos un formato diseñado sólo para la pesquisa en expectoración (Figura 3).

\section{5) Programas de crónicos}

En el SSMSO la mayoría de los casos pulmonares demostrados por baciloscopía durante el año 2004 estaba ya en control en alguno de los programas del adulto (Figura 4) por otras patologías. Es posible que esta situación se esté dando en otros servicios.

\section{6) La georeferencia o la desigualdad territo- rial en Tuberculosis}

Es conocido lo heterogénea que resulta la distribución de la tuberculosis en las poblaciones. Hay que definir qué haremos cuando identifiquemos las villas, poblaciones, barrios y campamentos, con mayor número de casos; si sostendremos actividades masivas, seguimientos familiares o trabajos conjuntos con los municipios.

Deberemos disponer de un sistema georeferencial para determinar la concentración puntual de los casos existentes y su movimiento histórico, para conocer la penetración, crecimiento o reducción de los casos de tuberculosis según su distribución territorial.

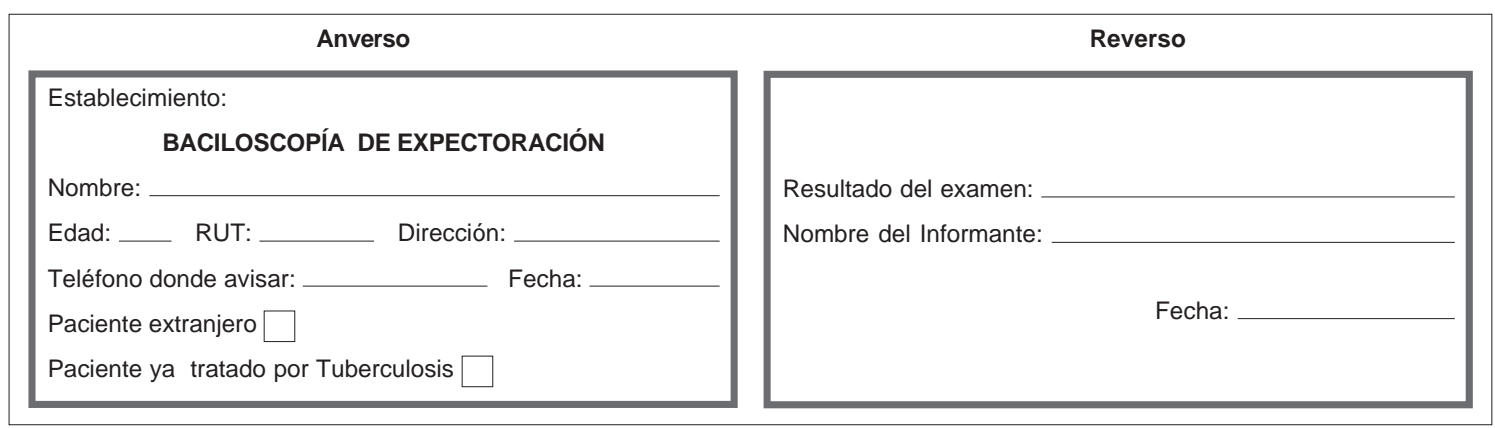

Figura 3. Formato simplificado de la orden de baciloscopía en expectoración. 


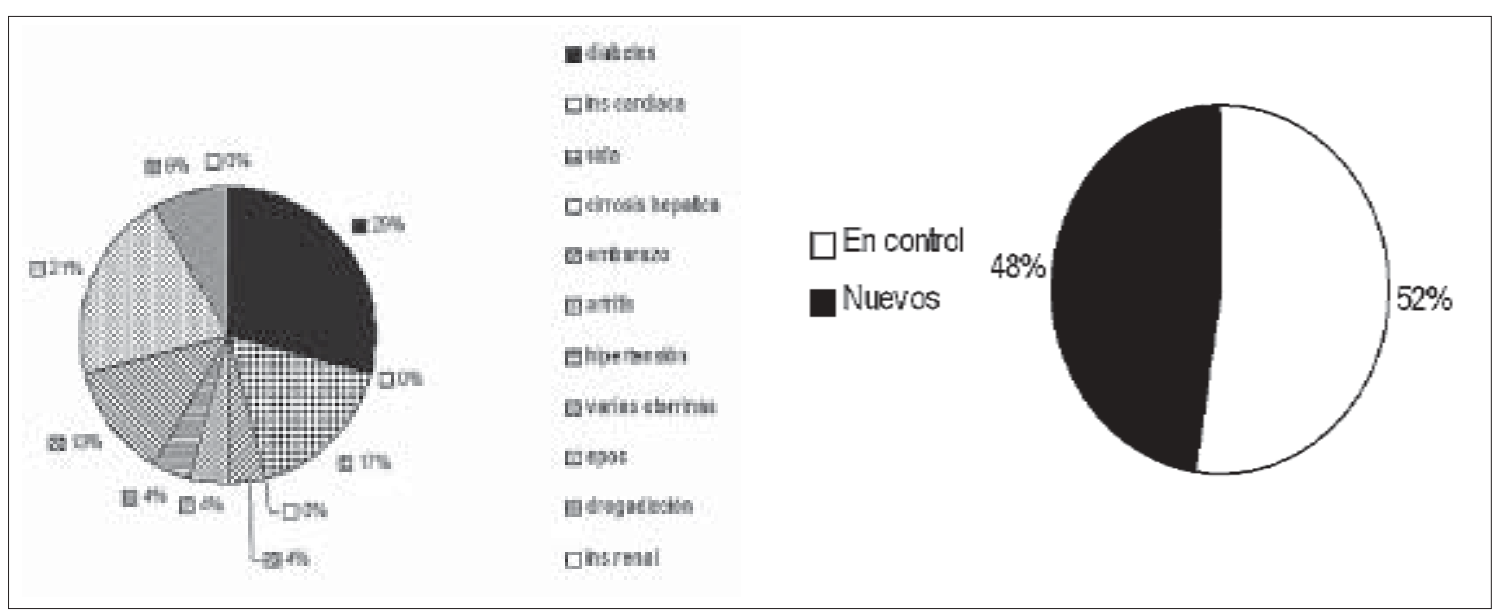

Figura 4. Tuberculosos pulmonares con enfermedades preexistentes bajo control médico regular al momento del diagnóstico $(\mathrm{n}=82)$. OTL = otorrinolaringológicas; $\mathrm{I}=$ insuficiencia.

\section{Bibliografía}

1.- MARRERO A, CAMINERO J A, RODRÍGUEZ R, BILLO N E. Towards elimination of tuberculosis in a low income country: the experience of Cuba 19621997. Thorax 2000; 55: 39-45.

2.- SAFER M A, THARPS Q J, JACKSON T C, LEVENTHAL H. Determinants of the three stages of delay in seeking care at a medical clinic. Med Care 1979; 17: 11-29.

3.- WEISSMAN J S, STERN R, FIELDING S L, EPSTEIN A M. Delayed access to health care: risk factors, reasons and consequences. Ann Intern Med 1991; 114: 325-31.

4.- RILEY R L, MOODIE A S. Infectivity of patients with pulmonary tuberculosis in inner homes. Am Rev Respir Dis 1974; 110: 810-2.

5.- WHO CDS TB/285. Involving private practitioners in tuberculosis control: issues, interventions and emerging policy framework. Genove. Document 2001

6.- SUÁREZ P G, WATT C J, ALARCÓN E, PORTOCARRERO J, ZAVALA D, CANALES R, et al. The dynamics of tuberculosis in response to 10 years of intensive control effort in Perú. J Infect Dis 2001; 184: 473-8.

7.- TAYLOR Z. 2004 What is the epidemiological impact of treatment latent TB infection? Toman's Tuberculosis 226-9.

9.- PEÑA C. Seminario Nacional de Evaluación de la Tuberculosis (2006). Programa Nacional de Tuberculosis. Olmué 2007.
Correspondencia a:

Dr. Fernando Tabilo Parraguez

Servicio de Medicina, Hospital Sótero del Río y

Programa de Control de la Tuberculosis, SSMSur-Oriente.

E-mail: ftabilo@ssmso.cl 